\title{
Neuroinflammation imaging markers for epileptogenesis
}

\author{
Tallie Z. Baram \\ Epilepsia, 58(Suppl. 3):11-19, 2017 \\ doi: 10.1111/epi.13778
}

*Matthias J. Koepp, †Eric Årstad, łens P. Bankstahl, §『Stefanie Dedeurwaerdere, \#**

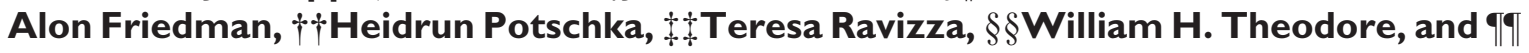

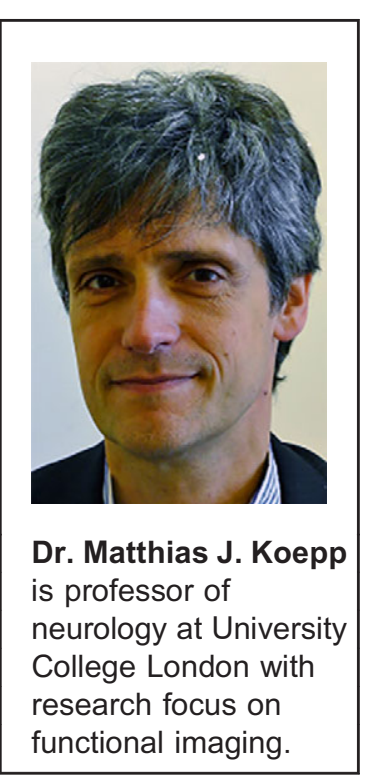

\begin{abstract}
SUMMARY
Epilepsy can be a devastating disorder. In addition to debilitating seizures, epilepsy can cause cognitive and emotional problems with reduced quality of life. Therefore, the major aim is to prevent the disorder in the first place: identify, detect, and reverse the processes responsible for its onset, and monitor and treat its progression. Epilepsy often occurs following a latent period of months to years (epileptogenesis) as a consequence of a brain insult, such as head trauma, stroke, or status epilepticus. Although this latent period clearly represents a therapeutic window, we are not able to stratify patients at risk for long-term epilepsy, which is prerequisite for preventative clinical trials. Moreover, because of the length of the latent period, an early biomarker for treatment response would be of high value. Finally, mechanistic biomarkers of epileptogenesis may provide more profound insight in the process of disease development. KEY WORDS: Epileptogenesis, Magnetic resonance imaging (MRI), Nuclear medicine imaging (PET/SPECT), Glia, Blood-brain barrier.
\end{abstract}

A key requirement for preventing epilepsy is to predict which individual will develop epilepsy prior to the onset of spontaneous seizures. This requires quantifiable, noninvasive, clinically relevant, reproducible and easy-to-perform markers with high sensitivity and specificity, which enable us to monitor the disease progress and the effects of

\footnotetext{
Accepted April 6, 2017.

*Institute of Neurology, University College London, London, United Kingdom; †Department of Chemistry and Institute of Nuclear Medicine, University College London, London, United Kingdom; \$Department of Nuclear Medicine, Hannover Medical School, Hannover, Germany; §University of Antwerp, Wilrijk, Belgium; qUCB Biopharma, Braine l'Alleud, Belgium; \#Ben-Gurion University of the Negev, Beer-Sheva, Israel; **Dalhousie University, Halifax, Nova Scotia, Canada; ††Institute of Pharmacology, Toxicology, and Pharmacy, Ludwig-MaximiliansUniversity, Munich, Germany; tDDepartment of Neuroscience, IRCCSInstitute for Pharmacological Research Mario Negri, Milan, Italy; §§NIH, Bethesda, Maryland, U.S.A.; and IIDepartments of Pediatrics, Anatomy/ Neurobiology, Neurology, University of California-Irvine, Irvine, California, U.S.A.

Address correspondence to Tallie Z. Baram, Departments of Pediatrics, Anatomy/Neurobiology, Neurology, University of California-Irvine, Irvine, CA, U.S.A. E-mail: tallie@uci.edu

Wiley Periodicals, Inc.

(C) 2017 International League Against Epilepsy
}

interventions. Imaging methodologies meet these requirements, and are thus suitable for predicting or early diagnosing epilepsy, in particular, drug-resistant epilepsy, and the effect of interventions aimed at modulating its development and progression (Fig. 1).

Imaging methodologies allow visualization of molecules and pathways involved in the mechanisms of the onset or progression of the epileptogenic process. ${ }^{1}$ A variety of imaging modalities are available, and imaging strategies and tracers are being developed to address these issues.

Numerous studies have shown that the recovery of brain function after injury is based on brain plasticity and remodeling of functional organizations. ${ }^{2}$ Although brain plasticity has been considered essential for recovery, there are also concerns related to eventual negative consequences. Aberrant plasticity has been suggested to be a crucial process in epileptogenesis after injury. Investigation of brain inflammation seems an ideal biomarker candidate as it is a key repair mechanism after brain insult and has been recognized as an important factor in the pathology of various types of epilepsy. 


\section{Key Points}

- Imaging methodologies allow visualization of molecules and pathways mechanistically involved in epileptogenesis

- MRI and nuclear medicine imaging (positron emission tomography/single-photon emission computed tomography [PET/SPECT]) methodologies can be used for imaging brain inflammatory processes

- Preclinical and clinical studies need to critically assess the validity of these imaging biomarkers of epileptogenesis and of drug resistance

The current state-of-the-art for imaging inflammation and inflammatory processes using preclinical and clinical magnetic resonance imaging (MRI) and nuclear medicine imaging (positron emission tomography/single-photon emission computed tomography [PET/SPECT]) methodologies are summarized here by describing the current cuttingedge methodologies and pointing out future directions.

\section{IMAgING MiCROVASCULAR Pathology and ELECTROPHYSIOLOGIC BIOMARKERS FOR EPILEPTOGENESIS}

\section{(Alon Friedman and Daniela Kaufer)}

In the field of brain protection and therapeutics, phase III clinical trials have repeatedly failed to show therapeutic efficacy. This has necessitated a reexamination of translational research in this area. A growing consensus holds, that in addition to better understanding of underlying disease mechanisms, there is a need for biomarker-driven strategies to (1) identify patients with specific brain pathobiologic processes that increase the risk for a brain disorder, (2) confirm engagement of the proposed molecular target by the therapeutic agent, and (3) provide quantitative measures to assess therapeutic efficacy. The goal of biomarker research is thus to facilitate clinical studies by enabling rationalized subject selection, dosing, timing, and duration of treatment. In the field of postinjury epilepsy (PIE), no such biomarker is currently available.

Microvascular pathology with associated blood-brain barrier (BBB) dysfunction and neuroinflammation was shown to underlie long-lasting dysfunction of the neurovascular unit and was associated with epileptogenesis. To identify these early pathobiologic-relevant disease-underlying processes, we developed MRI protocols as well as image and signal analysis methods to quantitatively follow microvascular pathology, specifically leaky BBB and electrophysiologic features of network dysfunction. Postmortem immunohistochemistry was used to confirm the spatial co-localization of imaging-based evidence of a leaky BBB to the presence of serum proteins (albumin and immunoglobulin $\mathrm{G}[\mathrm{IgG}]$ ) and neuroinflammation (activated astrocytes and microglia). In vivo electrocorticography was used to objectively and quantitatively identify alterations in network functions and the development of seizures in experimental rodents. ${ }^{3}$

Recent studies show that contrast-enhanced MRI (CEMRI) can be used noninvasively to quantitatively assess BBB pathology in different brain regions. As such, animal

Subjects exposed to a potential epileptogenic insult
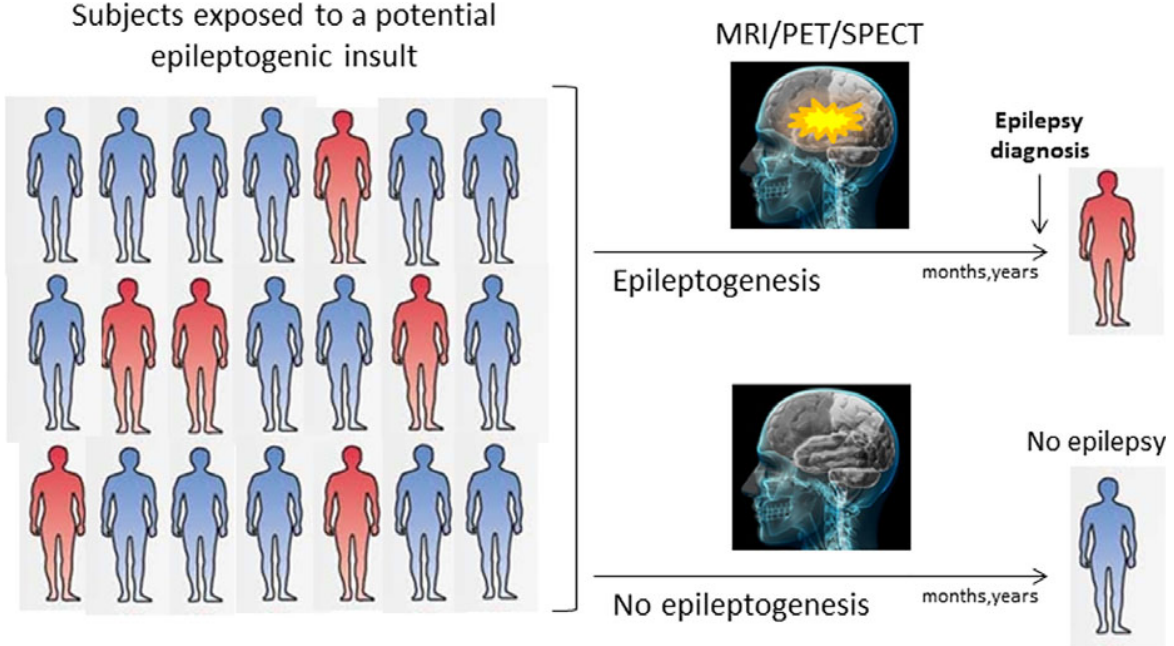

Figure I,

The role of imaging for monitoring epileptogenesis. A key requirement for preventing epilepsy is to predict which individual will develop epilepsy prior to the onset of spontaneous seizures. This requires quantifiable, noninvasive, clinically relevant, reproducible and easy-toperform markers with high sensitivity and specificity, which enable us to monitor the disease progress and the effects of interventions. Imaging methodologies meet these requirements, and are thus suitable for predicting or early diagnosing of epilepsy, in particular, drugresistant epilepsy, and the effect of interventions aimed at modulating its development and progression.

Epilepsia (c) ILAE 
experiments indicate that leaky BBB in limbic brain regions (specifically the piriform cortex, but also the amygdala and hippocampus) may serve as a sensitive and specific predictor (area under the curve [AUC] of 0.96, p < 0.0001) for epilepsy, whereas diffuse BBB breakdown (especially if includes the thalamus and septal nucleus) is associated with a lower risk. ${ }^{4}$ Brain regions with a pathologic leaky BBB were also showing a neuroinflammatory response, including the activation of astrocytes and microglia producing and releasing inflammatory mediators. Therefore, imaging of $\mathrm{BBB}$ breakdown may reflect the local presence and extent of inflammation. ${ }^{5}$ The advantage of CE-MRI is the potentially easy implementation in patients. Indeed, CE-MRI was shown to detect microvascular pathology (dysfunctional $\mathrm{BBB}$ ) in cortical regions surrounding contusional brain lesions following traumatic brain injury or stroke. Furthermore, these brain regions were associated with pathologic brain activity. These results suggest that identifying BBB pathology in cortical brain regions indicates a local neuroinflammatory response to injury that lowers seizure threshold and promotes epileptogenesis.

\section{Future directions}

Combining imaging with electrophysiologic recordings may determine whether brain tissue is undergoing epileptogenesis. Animal studies should confirm that antiinflammatory treatments are associated with both normalization of imaging and electrophysiologic alterations during epileptogenesis and a significant reduced risk for epilepsy.

\section{MRI $\mathbf{T}_{2} / \mathbf{T}_{2} *$ Signal Changes: A} Noninvasive, EARly and Reliable Predictive Marker of Temporal LOBE EPILEPSY AFTER FEBRILE Status EPIlepticus, With Translational Potential

\section{(Tallie Z. Baram)}

Temporal lobe epilepsy (TLE), with its associated cognitive and emotional problems is among the most common epilepsies, and is typically preceded by antecedent insults including trauma, brain infection, or prolonged childhood seizures. ${ }^{6,7}$ Indeed, prolonged febrile seizures, especially those longer than 30 min (febrile status epilepticus; FSE) precede $30-70 \%$ of individuals with TLE, and epilepsy develops in $\sim 40 \%$ of children with FSE. ${ }^{8-10}$ However, it is not possible to identify early which FSEexperiencing individuals will develop TLE years later, a crucial prerequisite for prevention of the spontaneous seizures and the comorbid cognitive and emotional impairments. Thus there is an unmet need for noninvasive, reliable and early predictive markers of epileptogenesis in individuals with FSE. ${ }^{1,11}$
We developed and characterized an immature rodent model of FSE (experimental FSE; eFSE). The eFSE is induced in rats on postnatal days 10-11, an age when hippocampal development approximates that of human infants-toddlers. ${ }^{12}$ In this model, hyperthermia is used, because fever cannot be generated in infant rats. ${ }^{10}$ However, the brain hyperthermia results in recruitment of fever mediators within the brain, and these are required for the generation of the eFSE, as shown through the use of transgenic mice lacking components of the interleukin 1 (IL-1) pathway. ${ }^{13}$ Whereas all rats develop eFSE in this model, these early life seizures generate TLE in only $33-40 \%$ of rats after a "silent period," analogous to the frequency and timecourse in humans. ${ }^{14}$

MRI is a clinically useful, noninvasive, repeatable tool. We employed high-field (11.7T) MRI providing resolution of limbic (temporal lobe) brain structures to probe eFSEinduced signal changes that might predict limbic epilepsy (TLE) development. ${ }^{1,14,15} \mathrm{We}$ then recorded long-term video-electroencephalography (EEG) to identify rats that become epileptic, and examine the neurobiologic basis of signal changes using neurochemical and neuroanatomic approaches.

Using a high-field, high-resolution (11.7T) MR scanner, reduction of $\mathrm{T}_{2}$ relaxation times in hippocampus and amygdala were noted in eFSE rats compared with normothermic and hyperthermic controls. ${ }^{1}$ It is notable that, $\mathrm{T}_{2}$ values in amygdala distinguished eFSE rats that became epileptic from eFSE rats that did not develop spontaneous seizures. The predictive signal was apparent already at $2 \mathrm{~h}$ after the eFSE and persisted at $6 \mathrm{~h}$ after the insult. The observed reduction of $\mathrm{T}_{2}$ values likely represented paramagnetic susceptibility effects $\left(\mathrm{T}_{2}{ }^{*}\right)$ derived from increased unsaturated venous hemoglobin, suggesting augmented oxygen utilization after FSE termination. Indeed, the use of deoxyhemoglobin-sensitive $\left(\mathrm{T}_{2}{ }^{*}\right)$ MRI sequences enabled visualization of the predictive changes on lower-field, clinically relevant MRI scanners (Fig. 2).

Looking for the origin of the high-oxygen and energy demanding processes that led to the high levels of deoxyhemoglobin, we focused on inflammation, because it is intrinsically required for febrile seizures and eFSE, ${ }^{13-18}$ is involved in epileptogenesis that follows eFSE, ${ }^{17,18}$ and is associated with high energy use. Searching for an early step in neuroinflammation, we found that the $T_{2}$ signal changes correlated with energy-demanding intracellular translocation of the injury-sensor HMGB1, a trigger of inflammatory cascades implicated in epileptogenesis. ${ }^{1,17-}$ 19 Accordingly, individual rats had major variance in $\mathrm{T}_{2}$ signal changes, and these correlated with HMGB1 nucleus-to-cytoplasm translocation, a prerequisite for its cellular release, and the expression of several key inflammatory mediators in the same rat. ${ }^{18} \mathrm{~T}_{2}{ }^{*}$ signal changes hold significant promise for identifying the risk for epilepsy in children experiencing eFSE. ${ }^{1,8}$ 


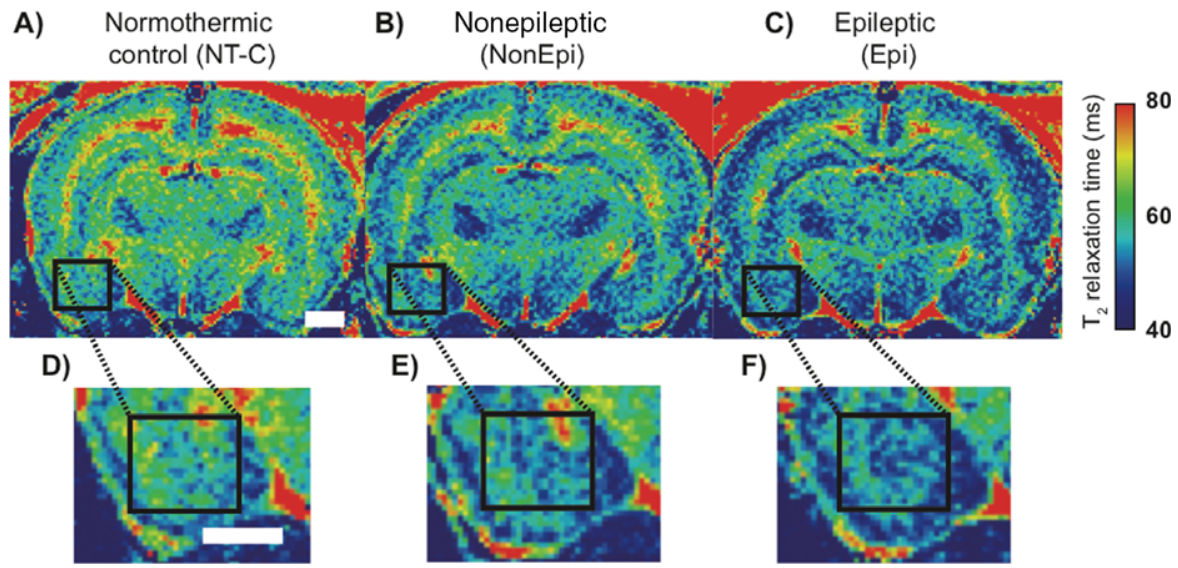

Figure 2.

A noninvasive magnetic resonance imaging (MRI) signal predicts the eventual development of epilepsy in an at-risk population. Top row: Representative color-coded quantitative $\mathrm{T}_{2}$ maps of a normothermic control (NT-C), as well as nonepileptic and epileptic rats experiencing experimental febrile status epilepticus (eFSE). The basolateral amygdala is highlighted in the black box. Bottom row: magnified views of the amygdalae. Scale bar indicates $\mathrm{I} \mathrm{mm}$. Measuring $\mathrm{T}_{2}$ values in the basolateral amygdala on the side with the lower $T_{2}$ relaxation time distinguished the epileptic group from the nonepileptic group, indicating that amygdala $T_{2}$ values were predictive of epilepsy. MRI scans were obtained $2 \mathrm{~h}$ after the end of the eFSE ( $3 \mathrm{~h}$ after its onset). An II.7T scanner was used, and the apparent reduction in $\mathrm{T}_{2}$ values was a result of the $T_{2} *$ effect.' Notably, the same predictive signal change can be seen in clinical-strength MR scanners when appropriate sequences are used.

Epilepsia (c) ILAE

\section{Multi-Modal Imaging of Vascular Cell Adhesion MOLECULE I (VCAM-I) EXPRESSION in Models of NeURoinflammation AND EPILEPSY}

\section{(Eric Arstad)}

Vascular cell adhesion molecule 1 (VCAM-1) is a member of the immunoglobulin supergene family that mediates the rolling and extravasation of leukocytes across the vascular endothelium. This is an inflammatory event that has been implicated in the progression of epilepsy in the lithium-pilocarpine mouse model. ${ }^{20}$ The development of micron-sized particles of iron oxide (MPIOs) coated with VCAM-1 targeting antibodies as a contrast agent (CA) for imaging VCAM1 expression with MRI has enabled neuroinflammation to be detected and localized in animal models of epilepsy. ${ }^{21}$ To identify the potential of SPECT for imaging of neuroinflammation, and to explore the value of multimodal imaging, VCAM-1 targeting MPIOs were labeled with iodine- $125^{22}$ Radiolabeling of the antibody-coated particles was straightforward and proceeded in high radiochemical yields using commercially available iodination tubes. In healthy rats, following injection into the right external jugular vein, $\left[{ }^{125} \mathrm{I}\right]$ VCAM-MPIO displayed rapid kinetics and was effectively cleared from the blood pool $2 \mathrm{~min}$ postinjection. Within $10 \mathrm{~min}$, the $\mathrm{CA}$ accumulated in the lungs ([mean $\pm \mathrm{SD}$ ] $4.8 \pm 1.3 \%$ injected dose [ID]/g), spleen $(3.4 \pm 1.4 \% \mathrm{ID} / \mathrm{g})$ and liver $(1.5 \pm 0.5 \%$ injected dose $[\mathrm{ID}] / \mathrm{g})$, with negligible uptake in the brain $(0.016 \pm 0.005 \% \mathrm{ID} / \mathrm{g})$. Low radioactivity levels in the bladder and thyroid suggest that $\left[{ }^{125}\right.$ I]VCAM-MPIO remains largely intact after administration in vivo. In (tumor necrosis factor $\alpha$ (TNF- $\alpha$ )-treated rats, the hypointensities present on MRI were in good agreement with the SPECT signals in the brain, and correlated well with phosphorimaging of cryosections from the site of TNF$\alpha$ injection. Quantification of both MRI and SPECT signals revealed higher CA binding in TNF- $\alpha$-treated animals compared to the two control groups, globally as well as in each of the brain hemispheres. Phosphorimaging of brain cryosections revealed a highly heterogeneous distribution, with the CA predominately confined to large blood vessels. The lithium-pilocarpine model of status epilepticus (SE) was used to further evaluate the properties of $\left[{ }^{125} \mathrm{I}\right]$ VCAM-MPIO as a CA. Status epilepticus with tonic-clonic seizures was induced 40-50 min after pilocarpine administration, and the seizures were terminated $90 \mathrm{~min}$ after SE onset by administration of diazepam. The animals were imaged approximately $20 \mathrm{~h}$ later (Fig. 3). SPECT/CT revealed high localized uptake of the CA in the cerebellum, cortex, brainstem, and olfactory bulb. With MRI, hypointensities were observed mainly near the hippocampus and ventricles, but also in the cerebral cortex and hypothalamic regions. Phosphorimaging of coronal brain cryosections confirmed high uptake of $\left[{ }^{125} \mathrm{I}\right] \mathrm{VCAM}-$ MPIO in the cortex, hippocampus, and hypothalamus. As seen for the TNF- $\alpha$-treated animals, $\left[{ }^{125}\right.$ I]VCAM-MPIO distributed predominantly to larger blood vessels.

\section{Future directions}

Further work will determine how faithfully this reflects the distribution of the VCAM-1 receptor in the brain's 


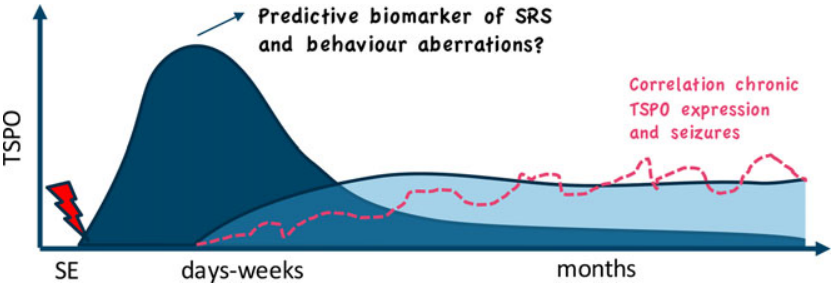

Figure 3.

Overview of TSPO upregulation during SE-induced epileptogenesis. TSPO upregulation is hypothesized to result from two superimposed processes related to the response to SE on the one hand and spontaneous recurrent seizures. Because some studies have shown a correlation between seizures and TSPO expression, it may represent a seizure biomarker. In addition, TSPO PET may act as a theragnostic marker for anti-inflammatory treatment. Future studies will investigate whether early inflammation could be a biomarker of development of epilepsy and comorbidities.

Epilepsia (C) ILAE

pathologic state or whether this phenomenon might to some extent be due to changes in blood flow. We are currently developing a peptide-derived VCAM-1 nuclear imaging agent to overcome some of the limitations of MPIOs, and potentially allow translation to human studies.

\section{IMAGING ASTROCYTES DURING Epileptogenesis by Proton Magnetic Resonance SPECTROSCOPY}

\section{(Teresa Ravizza, Annamaria Vezzani)}

The activation of astrocytes is one of the most prominent cellular changes occurring in the brain after various epileptogenic injuries. Studies in experimental models of epilepsy showed that activated astrocytes produce and release an array of proinflammatory mediators during epileptogenesis that potentially contribute to spontaneous seizures generation, ${ }^{23}$ cell loss, and comorbidity. ${ }^{24}$ Because of the potential mechanistic role of neuroinflammation in epilepsy, monitoring this process using noninvasive approaches may generate potential biomarkers of ictogenesis and epileptogenesis.

Proton magnetic resonance spectroscopy $\left({ }^{1} \mathrm{H}-\mathrm{MRS}\right)$ is a noninvasive in vivo imaging technique that allows the detection and quantification of brain metabolites of which myo-Inositol (mIns) specifically reflects the functional activation of astrocytes. ${ }^{25} \mathrm{We}$ designed therefore an in vivo longitudinal study to assess hippocampal mIns levels in a rat SE model of epilepsy where spontaneous seizures develop in $100 \%$ of animals. We found that mIns levels increase progressively during epileptogenesis. ${ }^{26}$ Notably, these changes occurred before the onset of spontaneous seizures with mIns levels maintained as high as before epilepsy onset in chronic epileptic rats. A positive correlation was found between hippocampal mIns levels in epileptic rats and immunohistochemical Glial fibrillary acid protein (GFAP) expression, thus confirming the association between changes in mIns and astroglial activation. ${ }^{26}$ Receiver-operating characteristic (ROC) analysis showed that mIns levels measured before epilepsy onset differentiate with high fidelity $(\mathrm{AUC}=1.0)$ control rats from rats that develop epilepsy. We further studied mIns as a potential biomarker of epileptogenesis using an experimental models of brain injury, where epilepsy develops only in a subpopulation of animals. By inducing SE in postnatal day (PN)21 rats with lithium+pilocarpine, only $60-70 \%$ of animals develop epilepsy, although SE severity and duration are similar in all rats. ${ }^{1} \mathrm{H}-\mathrm{MRS}$ analysis was done longitudinally post-SE at selected times before spontaneous seizures arose. Epileptic rats were identified by the presence of spontaneous videoEEG seizures and decreased hippocampal afterdischarge threshold assessed 7 months post-SE, and were distinguished from nonepileptic rats showing no spontaneous seizures and with a threshold similar to that of sham controls (not exposed to SE). mIns levels were retrospectively analyzed and compared in these two cohorts of rats. We found that mIns hippocampal levels were persistently increased post-SE only in rats prone to develop epilepsy. ${ }^{27}$ ROC analysis of mIns levels at the predicted time of disease onset showed a good discrimination between these two rat cohorts (AUC $=0.83$ ).

These results provide a proof-of-principle demonstration that mIns levels following potential epileptogenic injuries may represent a clinically meaningful biomarker for early identification of individuals at risk for developing epilepsy.

\section{Future directions}

${ }^{1} \mathrm{H}-\mathrm{MRS}$ is an imaging approach previously used for detecting astroglia activation in human epilepsy (i.e., TLE, tuberous sclerosis, and Rasmussen's encephalitis). Further studies are warranted for validating mIns as a biomarker of epileptogenesis in the clinical setting. For the same purpose, PET/SPECT detection of astrogliosis using tracers targeting monoaminoxidase-B (MAO), an enzyme preferentially localized in astrocytes, should be considered. Notably, an increased uptake of ${ }^{11} \mathrm{C}$-deuterium-deprenyl, a MAO inhibitor, has been described in the hippocampus of patients with TLE, which was associated with astrogliosis assessed by ${ }^{3} \mathrm{H}$-L-deprenyl autoradiography. ${ }^{28-30}$ Similar findings were reported using the SPECT tracer ${ }^{123}$ I-Ro43-0463. ${ }^{31}$

\section{Imaging Glia by Positron EMISSION TOMOGRAPHY (PET) AS A BIOMARKER OF EPILEPTOGENESIS}

\section{(Stefanie Dedeurwaerdere, Jens P. Bankstahl)}

Molecular imaging with PET and specific radioligands for translocator protein $18 \mathrm{kDa}$ (TSPO) offer the possibility to visualize in vivo the cellular inflammatory processes in 
the brain, and more particularly microglia activation during epileptogenesis. ${ }^{32}$ To be considered a useful biomarker for studying a pathologic condition, TSPO expression has to be altered during the disease. In this regard, longitudinal in vivo TSPO PET imaging during epileptogenesis in the lithium+pilocarpine SE rat model revealed increased microglia activation in epileptogenesis-associated brain regions within days from the acute insult and peaking 1-2 weeks after SE. ${ }^{33,34}$ This evidence was replicated in the systemic and focal intrahippocampal kainic acid post-SE rodent mod$\mathrm{els}^{32}$ and is in accordance with histologic assessment of microglia activation and the time course of neuroinflammatory markers measured with histologic or biochemical techniques. In vivo imaging data were also validated by measuring TSPO autoradiographic binding postmortem in the same animals.

To appreciate the value of TSPO PET as a prognostic biomarker of epilepsy, we need to evaluate its relation to spontaneous seizures. Recently, several studies in rat SE models have described a relationship between seizures and increased TSPO expression. First, it was observed that a severe SE results in a stronger TSPO upregulation compared to a mild SE. ${ }^{35}$ Secondly, TSPO expression in hippocampus and extrahippocampal temporal lobe regions in the chronic phase of epilepsy positively correlates with the frequency of spontaneous seizures. ${ }^{32,36}$ Figure 4 represents a graphical summary of the temporal evolution of TSPO expression and its potential relationship to behavioral phenotypes such as comorbidities and spontaneous seizures in the SE models.

Recent work provided proof-of-concept for an association between TSPO expression measured with the translocator protein ligand ${ }^{18} \mathrm{~F}-\mathrm{PBR} 111$ PET and behavioral comorbidities related to depression-like symptoms in the rat kainic acid post-SE model. ${ }^{37}$ Moreover, when applying a multivariate modeling approach, the TSPO PET scans at disease onset ( 2 weeks after SE) were predictive of the

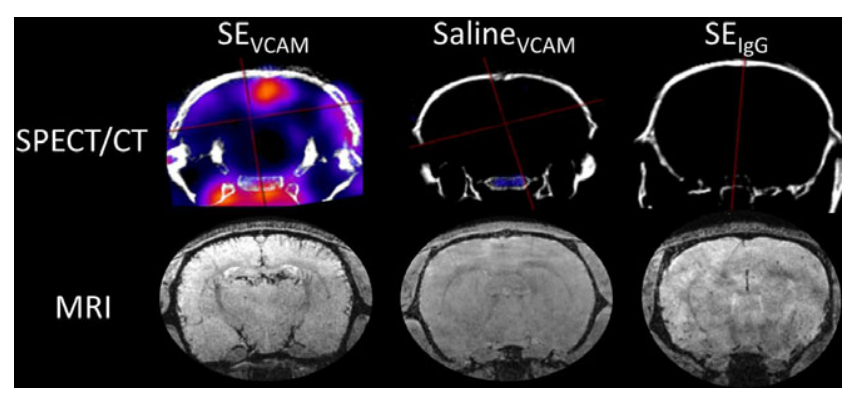

Figure 4.

In vivo imaging of neuroinflammation in the lithium-pilocarpine model of SE with [ ${ }^{125}$ I]VCAM-MPIO. The distribution of the CA was assessed by SPECT/CT (top row) and MRI (bottom row). Images from an SE rat (left), a sham-treated control rat (middle), and an SE rat administered with immunoglobulin-coated $\left[{ }^{125}\right]$ IMPIO CA (right).

Epilepsia (c) ILAE individual seizure burden of the epileptic animals. These data indicate that TSPO PET scans could be useful for prognosis of epilepsy severity at disease onset. However, these results remain to be reproduced by independent groups and translated to other animal models. In addition, investigations of the theragnostic value of TSPO PET after anti-inflammatory treatment are warranted because first attempts showed detectable, although moderate, decrease in TSPO signal. ${ }^{38}$ Currently, TSPO-based PET imaging limited to the investigation of neuroinflammation processes linked to overexpression of TSPO. Alternatively, it would be valuable to investigate PET ligands visualizing different players in brain inflammation when available.

\section{IMAGING OF GLIA AS A BIOMARKER OF DRUG RESISTANCE}

\section{(Heidrun Potschka)}

Drug resistance in epilepsy is considered a multifactorial phenomenon. Strong evidence exists that disease-associated molecular and cellular alterations can affect both the pharmacokinetics and pharmacodynamics of antiepileptic drugs. ${ }^{39}$ Experimental studies indicate that excessive inflammatory signaling may have an impact on BBB penetration of antiepileptic drugs or their interaction with target sites. Moreover, inflammatory mediators play a role in neuronal hyperexcitability and ictogenesis, thereby contributing to intrinsic severity of epilepsy. ${ }^{40,41}$

TSPO has repeatedly been described as a molecular marker of microglial activation. Even though TSPO is also expressed in other cell types, its expression was proven to correlate with activation of microglia in several studies. ${ }^{42}$ Therefore, we have hypothesized that molecular imaging of microglia activation using a radioligand of TSPO might serve as a biomarker for pharmacoresistance. To address this hypothesis, groups of phenobarbital responder and nonresponder rats have been selected in the chronic epilepsy phase of a post-SE model and subjected to micro-PET analysis. In rats with drug-resistant seizures, $(R)-\left[{ }^{11} \mathrm{C}\right] \mathrm{PK} 11195$ brain uptake values in the hippocampus, caudate putamen, parietal cortex, and occipital cortex significantly exceeded that in electrode-implanted control rats. ${ }^{36}$ Notably, data from rats with a relevant pharmacoresponse were proven to be in the control range.

These findings suggest that TSPO PET imaging might serve as a biomarker of drug resistance. However, it needs to be carefully assessed in future studies whether the correlation between brain uptake of TSPO radioligands and pharmacoresponsiveness does not merely reflect seizure frequency. Because experimental data did not indicate that setting a cut-off would reliably distinguish responders from nonresponders, it remains doubtful that this imaging approach would reach the required specificity and sensitivity for clinical translation. 


\section{Future directions}

Data obtained with $(R)-\left[{ }^{11} \mathrm{C}\right] \mathrm{PK} 11195$ in responders suggest that this radiotracer is not optimal for imaging of epilepsy development. Thus the novel TSPO tracer $\left[{ }^{18} \mathrm{~F}\right] \mathrm{GE} 180$, with superior affinity and selectivity, should be used for longitudinal micro-PET studies for monitoring the tracer uptake during epileptogenesis and exploring its predictive value.

To identify novel biomarkers of neuroinflammation that may be exploited for imaging studies, we have completed a liquid chromatography-mass spectrometry (LC/MS)-based proteomic analysis of hippocampal as well as parahippocampal cortex tissue sampled at different time points during epileptogenesis in a rat SE model. Bioinformatic analysis provides information about temporal changes in protein expression of inflammatory regulatory pathways. ${ }^{43}$ We are currently analyzing these data with a systems biology approach and network analysis.

\section{PET IMAGING OF INFLAMMATION IN HuMAN EPILEPSY}

(William H. Theodore)

TSPO, previously known as the peripheral benzodiazepine receptor, is overexpressed in activated microglia and reactive astrocytes. The previous generation of PET TSPO ligands such as PK-11195 showed increased activity in patients with syndromes such as Rasmussen's encephalitis, but studies in patients with TLE and other focal syndromes were unrevealing. Several new ligands with better relative brain to peripheral binding have become available. In addition, a polymorphism leading to low brain uptake in about $10 \%$ of patients was discovered that can be tested in leukocytes.

In a preliminary study using $\left[{ }^{11} \mathrm{C}\right] \mathrm{PBR} 28$ in patients with mesial TLE, we found relatively increased binding ipsilateral to seizure foci. ${ }^{44} \mathrm{We}$ then wished to determine whether patients with TLE have widespread TSPO overexpression, and to investigate patients with neocortical and extratemporal foci using $\left[{ }^{11} \mathrm{C}\right] \mathrm{PBR} 28$ and another TSPO radioligand, $\left[{ }^{11} \mathrm{C}\right] \mathrm{DPA}-713$. Twenty-three patients with TLE and 11 age-matched controls were scanned with $\left[{ }^{11} \mathrm{C}\right] \mathrm{PBR} 28$, and eight patients and seven controls were scanned with $\left[{ }^{11} \mathrm{C}\right]$ DPA-713. A subset had arterial blood sampling for full quantification. Patients had unilateral seizure foci based on ictal EEG and structural MRI. Participants with homozygous low-affinity TSPO binding were excluded. ${ }^{45}$ The $\left[{ }^{11} \mathrm{C}\right]$ PBR28 distribution volume (VT) corrected for free fraction (fP) was measured in patients and controls using FreeSurfer software. Side-to-side asymmetry in patients with TLE was calculated as the ratio of ipsilateral to contralateral $\left[{ }^{11} \mathrm{C}\right]$ PBR28 and $\left[{ }^{11} \mathrm{C}\right] \mathrm{DPA}-713$ standardized uptake values from temporal regions. The $\left[{ }^{11} \mathrm{C}\right] \mathrm{PBR} 28 \mathrm{VT}$ to $\mathrm{fP}$ ratio was higher in patients with TLE than in controls for all ipsilateral temporal regions $(27-42 \%$; $<<0.05)$ and in contralateral hippocampus, amygdala, and temporal pole (approximately 30-32\%; $\mathrm{p}<0.05$ ). Individually, 12 patients, 10 with mesial temporal sclerosis, had asymmetrically increased hippocampal $\left[{ }^{11} \mathrm{C}\right] \mathrm{PBR} 28$ uptake exceeding the $95 \%$ confidence interval (CIs) of the controls. Binding of $\left[{ }^{11} \mathrm{C}\right] \mathrm{PBR} 28$ was increased significantly in thalamus. Relative $\left[{ }^{11} \mathrm{C}\right] \mathrm{PBR} 28$ and $\left[{ }^{11} \mathrm{C}\right] \mathrm{DPA}-713$ uptakes were higher ipsilateral than contralateral to seizure foci in patients with TLE ([ $\left.{ }^{11} \mathrm{C}\right]$ PBR28: 2-6\%; $\left[{ }^{11} \mathrm{C}\right] \mathrm{DPA}-713$ : 4-9\%). Asymmetry of $\left[{ }^{11} \mathrm{C}\right] \mathrm{DPA}-713$ was greater than that of $\left[{ }^{11} \mathrm{C}\right]$ PBR28 $(\mathrm{F}=29.4 ; \mathrm{p}=0.001) .{ }^{45}$

Nine patients with neocortical foci had $\left[{ }^{11} \mathrm{C}\right] \mathrm{PBR} 28$ PET scans. Four patients had surgical resections with pathologic findings of focal cortical dysplasia (FCD) type IIA and IIB. Preselected regions of interest (ROIs) were delineated using $\mathrm{T}_{1}$-weighted MRI and FreeSurfer based on patients' seizure foci. All nine patients had asymmetry indices that exceeded the $95 \% \mathrm{CI}$ of the control mean in at least one region related to the focus. Asymmetry did not differ based on the presence of FCD in surgical tissue samples. TSPO binding is increased both ipsilateral and contralateral to seizure foci in patients with TLE, suggesting ongoing inflammation. Notably, a recent study in a patient with refractory frontal epilepsy due to FCD type II confirmed that TSPO binding increased in the clinically defined seizure focus; moreover, the postseizure scan done at least 2 weeks after a seizurefree period showed persistent and more widespread signal, possibly reflecting seizure spread. ${ }^{46}$

\section{Future directions}

Future perspectives are to investigate whether there is a link between the peak in TSPO increase during the latent phase after an acute injury and the sequelae of seizure development and behavioral comorbidities in different models of acquired epilepsy. This information will help to guide the clinical application in patients exposed to potential epileptogenic injuries. It may be hypothesized that TSPO overexpression results from two potentially interrelated and overlapping processes resulting from the response to injury and the recurring spontaneous seizures (Fig. 4).

\section{Conclusions and Future DireCTIONS}

This review of preclinical and clinical biomarkers of epileptogenesis indicates that several innovative imaging methods offer promise of identifying predictors or correlates of neuroinflammation and other related cellular processes that portend or accompany epilepsy. Novel signatures delineate the onset of epileptogenic cascades, molecules involved in BBB dysfunction, neuroinflammation, and activation of glial cells, and all have promising translational potential for clinical use.

Broad future experimental and clinical approaches are noted here. First, as most of the methods are innovative and 
novel, studies are necessary to critically assess the validity of these imaging biomarkers of epileptogenesis and of drug resistance. Preclinical studies should evaluate predictive values with imaging prior to antiepileptic drug exposure and in models with comparison between subgroups with or without epilepsy manifestation following a brain insult. Clinical studies should be performed longitudinally, ideally comparing groups of patients who develop spontaneous seizures after similar injury with those that do not develop seizures over a similar time frame. Moreover, specificity and sensitivity need to be assessed carefully in different epilepsy models. In addition, efforts are necessary to identify alternate molecular markers and to validate their informative value in the context of development and pharmacoresistance of epilepsy.

Future studies are also needed to investigate the effect of disease-modifying treatment on the imaging biomarkers and potential subsequent seizure prevention as a strategy to evaluate treatment response.

\section{Acknowledgments}

Additional contributors to the studies described in this manuscript are the following: G Bar-Klei R Veksler, D Milikovsky (Ben-Gurion University of the Negev, Beer-Sheva, Israel); MC Curran, M Choy, CM Dubé, KP Patterson, A Obenaus (University of California-Irvine and Loma Linda University, CA, U.S.A.); N Patel, BA Duffy, A Badar, MF Lythgoe (Centre for Advanced Biomedical Imaging, University College London, United Kingdom); R Pascente, F Frigerio, M Filibian, M Rizzi, D Tolomeo (IRCCS-Mario Negri Institute for Pharmacological Research, Milano, Italy); L Gershen, P Zanotti-Fregonara, I Dustin, J Liow, J Hirvonen, SS Zoghbi, RB Innis (NIH, Bethesda, MD, U.S.A.).

\section{Disclosure}

We have no conflicts of interest with regard to this manuscript. We confirm that we have read the Journal's position on issues involved in ethical publication and affirm that this report is consistent with those guidelines.

\section{REFERENCES}

1. Choy M, Dube CM, Patterson K, et al. A novel, noninvasive, predictive epilepsy biomarker with clinical potential. $J$ Neurosci 2014;34:8672-8684.

2. Failla MD, Wagner AK. Models of posttraumatic brain injury neurorehabilitation. In Kobeissy FH (Ed) Brain neurotrauma: molecular, neuropsychological, and rehabilitation aspects. Boca Raton, FL: CRC Press/Taylor \& Francis, 2015: Chapter 35

3. Rizzi M, Weissberg I, Milikovsky DZ, et al. Following a potential epileptogenic insult, prolonged high rates of nonlinear dynamical regimes of intermittency type is the hallmark of epileptogenesis. Sci Rep 2016;6:31129.

4. Veksler R, Shelef I, Friedman A. Blood-brain barrier imaging in neuropathologies. Arch Med Res 2014;45:646-652.

5. Vezzani A, Friedman A. Brain inflammation as a biomarker in epilepsy. Biomark Med 2011;5:607-614.

6. Cendes F, Andermann F, Gloor P, et al. Atrophy of mesial structures in patients with temporal lobe epilepsy: cause or consequences of repeated seizures? Ann Neurol 1993;34:795-801.

7. Chong J, Hesdorffer DC, Thurman DJ, et al. The prevalence of epilepsy along the Arizona-Mexico border. Epilepsy Res 2013;105:206215 .
8. Annegers JF, Hauser WA, Shirt SB, et al. Factors prognostic of unprovoked seizures after febrile convulsions. N Engl J Med 1987;316:493498.

9. Cendes F, Andermann F, Dubeau F, et al. Early childhood prolonged febrile convulsions, atrophy and sclerosis of mesial structures, and temporal lobe epilepsy: an MRI volumetric study. Neurology 1993;34:1083-1087.

10. Dubé CM, Brewster AL, Richichi C, et al. Fever, febrile seizures and epilepsy. Trends Neurosci 2007;30:490-496.

11. Grohn O, Pitkanen A. Magnetic resonance imaging in animal models of epilepsy-noninvasive detection of structural alterations. Epilepsia 2007;48:3-10.

12. Avishai-Eliner S, Brunson KL, Sandman CA, et al. Stressed-out, or in (utero)? Trends Neurosci 2002;25:518-524.

13. Dubé C, Vezzani A, Behrens M, et al. Interleukin-1beta contributes to the generation of experimental febrile seizures. Ann Neurol 2005;57:152-155.

14. Dubé CM, Ravizza T, Hamamura M, et al. Epileptogenesis provoked by prolonged experimental febrile seizures: mechanisms and biomarkers. J Neurosci 2010;30:7484-7494.

15. Brennan GP, Dey D, Chen Y, et al. Dual and opposing roles of microRNA-124 in epilepsy are mediated through inflammatory and NRSFdependent gene networks. Cell Rep 2016;14:2402-2412.

16. Heida JG, Boisse L, Pittman QJ. Lipopolysaccharide-induced febrile convulsions in the rat: short-term sequelae. Epilepsia 2004;45:13171329.

17. Vezzani A, French J, Bartfai T, et al. The role of inflammation in epilepsy. Nat Rev Neurol 2011;7:31-40.

18. Patterson KP, Brennan GP, Curran B, et al. Coordinate inflammatory responses after experimental febrile status epilepticus: implication for epileptogenesis. eNeuro 2015;2:e0034-0015.

19. Iori V, Maroso M, Rizzi M, et al. Receptor for Advanced Glycation Endproducts is upregulated in temporal lobe epilepsy and contributes to experimental seizures. Neurobiol Dis 2013;58:102-114.

20. Fabene PF, Mora GN, Martinello M, et al. A role for leukocyteendothelial adhesion mechanisms in epilepsy. Nat Med 2008;14:13771383.

21. Duffy BA, Choy M, Riegler J, et al. Imaging seizure-induced inflammation using an antibody targeted iron oxide contrast agent. NeuroImage 2012;60:1149-1155.

22. Patel N, Duffy BA, Badar A, et al. Bimodal imaging of inflammation with SPECT/CT and MRI using iodine-125 labeled VCAM-1 targeting microparticle conjugates. Bioconjug Chem 2015;26:1542-1549.

23. Vezzani A. Anti-inflammatory drugs in epilepsy: does it impact epileptogenesis? Expert Opin Drug Saf 2015;14:583-592.

24. Mazarati A, Vezzani A. Inflammation and immunomodulation in epilepsy and its comorbidities. In Boison D, Masino SA (Eds) Homeostatic control of brain function. Oxford: Oxford University Press, 2015:155-174.

25. Brand A, Richter-Landsberg C, Leibfritz D. Multinuclear NMR studies on the energy metabolism of glial and neuronal cells. Dev Neurosci 1993;15:289-298.

26. Filibian M, Frasca A, Maggioni D, et al. In vivo imaging of glia activation using ${ }^{1} \mathrm{H}$-magnetic resonance spectroscopy to detect putative biomarkers of tissue epileptogenicity. Epilepsia 2012;53: 1907-1916.

27. Pascente R, Frigerio F, Rizzi M, et al. Cognitive deficits and brain myo-Inositol are early biomarkers of epileptogenesis in a rat model of epilepsy. Neurobiol Dis 2016;93:146-155.

28. Kumlien E, Hilton-Brown P, Spannare B, et al. In vitro quantitative autoradiography of [3H]-L-deprenyl and [3H]-PK 11195 binding sites in human epileptic hippocampus. Epilepsia 1992;33:610-617.

29. Kumlien E, Bergstrom M, Lilja A, et al. Positron emission tomography with [11C]deuterium-deprenyl in temporal lobe epilepsy. Epilepsia 1995;36:712-721.

30. Kumlien E, Nilsson A, Hagberg G, et al. PET with 11C-deuteriumdeprenyl and 18F-FDG in focal epilepsy. Acta Neurol Scand 2001;103:360-366.

31. Buck A, Frey LD, Bläuenstein P, et al. Monoamine oxidase B signlephoton emission tomography with [123]Ro 43-0463: imaging in volunteers and patients with temporal lobe epilepsy. Eur J Nucl Med 1998;25:464-470. 
32. Amhaoul H, Hamaide J, Bertoglio D, et al. Brain inflammation in a chronic epilepsy model: evolving pattern of the translocation protein during epileptogenesis. Neurobiol Dis 2015;82:526-539.

33. Brackhan M, Bascunana P, Postema JM, et al. Serial quantitative TSPO-targeted PET reveals peak microglial activation up to 2 weeks after an epileptogenic brain insult. $J$ Nucl Med 2016;57:1302-1308

34. Yankam Njiwa J, Costes N, Bouillot C, et al. Quantitative longitudinal imaging of activated microglia as a marker of inflammation in the pilocarpine rat model of epilepsy using [11C]-(R)-PK11195 PET and MRI. J Cereb Blood Flow Metab 2017;37:1251-1263.

35. Dedeurwaerdere S, Callaghan PD, Pham T, et al. PET imaging of brain inflammation during early epileptogenesis in a rat model of temporal lobe epilepsy. EJNMMI Res 2012;2:60.

36. Bogdanovic RM, Syvanen S, Michler C, et al. (R)-[11C]PK11195 brain uptake as a biomarker of inflammation and antiepileptic drug resistance: evaluation in a rat epilepsy model. Neuropharmacology 2014;85:104-112.

37. Bertoglio D, Verhaeghe J, Santermans E, et al. Non-invasive PET imaging of brain inflammation at disease onset predicts spontaneous recurrent seizures and reflects comorbidities. Brain Behav Immun 2017;61:69-79.

38. Airas L, Dickens AM, Elo P, et al. In vivo PET imaging demonstrates diminished microglial activation after fingolimod treatment in an animal model of multiple sclerosis. J Nucl Med 2015;56:305-310.
39. Potschka H. Animal and human data: where are our concepts for drugresistant epilepsy going? Epilepsia 2013;54(Suppl 2):29-32.

40. Vezzani A, Auvin S, Ravizza T, et al. Glia-neuronal interactions in ictogenesis and epileptogenesis: role of inflammatory mediators. In Noebels JL, Avoli M, Rogawski MA, Olsen RW, Delgado-Escueta AV (Eds) Jasper's basic mechanisms of the epilepsies. 4th Ed. Bethesda, MD: National Center for Biotechnology Information (US), 2012:618629.

41. Vezzani A, Viviani B. Neuromodulatory properties of inflammatory cytokines and their impact on neuronal excitability. Neuropharmacology $2015 ; 96: 70-82$.

42. Amhaoul H, Staelens S, Dedeurwaerdere S. Imaging brain inflammation in epilepsy. Neuroscience 2014;279:238-252.

43. Walker A, Russmann V, Deeg CA, et al. Proteomic profiling of epileptogenesis: focus on inflammation. Brain Behav Immun 2016;53:138158.

44. Hirvonen J, Kreisl WC, Fujita M, et al. Increased in vivo expression of an inflammatory marker in temporal lobe epilepsy. J Nucl Med 2012;53:234-240.

45. Gershen LD, Zanotti-Fregonara P, Dustin IH, et al. Neuroinflammation in temporal lobe epilepsy measured using positron emission tomographic imaging of translocator protein. JAMA Neurol 2015;72:882888 .

46. Butler T, Li Y, Tsui W, et al. Transient and chronic seizure-induced inflammation in human focal epilepsy. Epilepsia 2016;57:e191-e194. 